\title{
La privatización del asesoramiento agrícola: consecuencias para los productores lecheros del valle del Mantaro, Perú
}

Guy Faure*

Kary Huamanyauri Méndez**

Ivonne Salazar***

Carlos Gómez ${ }^{* * * *}$

Erwin de $\mathrm{Nys}^{* * * * *}$

Michel Dulcire******

doi:I0.11144/Javeriana.cdrı2-76.paac

Recibido: 2014-12-20 Aprobado: 2015-04-27 Disponible en línea: 2015-10-0I

Cómo citar este artículo: Fauré, G., Huamanyauri Méndez, K., Salazar, I., Gómez, C., de Nys, E., \& Dulcire, M. (2015). La privatización del asesoramiento agrícola: consecuencias para los productores lecheros del valle del Mantaro, Perú. Cuadernos de Desarrollo Rural, I2(76), II-33. http://dx.doi.org/ıo.III44/Javeriana.cdrı2-76.paac

* Investigador, socio-economía, Centre de Coopération Internationale en Recherche Agronomique pour le Développement, CIRAD, UMR Innovation. Correo electrónico: guy.faure@cirad.fr

** Ingeniero Zootecnista, Universidad Nacional Agraria La Molina, UNALM, Unidad de Extensión, Facultad de Zootecnia. Perú. Correo electrónico: khuamanyauri@lamolina.edu.pe

*** Docente, Universidad Nacional Agraria La Molina, UNALM. Departamento de Nutrición, Facultad de Zootecnia. Perú. Correo electrónico: sri@lamolina.edu.pe

**** Docente, Universidad Nacional Agraria La Molina, UNALM. Departamento de Nutrición, Facultad de Zootecnia. Correo electrónico: cagomez@lamolina.edu.pe

***** Especialista recursos hídricos, Banco Mundial, Departamento de América Latina y Caribe. Correo electrónico: edenys@worldbank.org

******* Investigador, sociología rural, Centre de Coopération Internationale en Recherche Agronomique pour le Développement, CIRAD, UMR Innovation. Correo electrónico: michel.dulcire@cirad.fr 


\title{
Resumen
}

A nivel mundial, el papel del sector privado en el asesoramiento agrícola ha venido incrementándose desde los años ochenta y noventa. Una muestra de tal evolución se observa en el valle del Mantaro (Perú), en un contexto de pequeña agricultura. Para evaluar sus consecuencias, se realizaron entrevistas con proveedores de insumos, extensionistas y productores. En este caso, el asesoramiento privado se realiza de manera asociada a la venta de insumos, lo cual, si bien favorece el acceso de un apoyo técnico a casi todos los productores, no considera sus necesidades globales. Esta investigación muestra la importancia de una mejor coordinación entre los actores públicos y privados del sistema de asesoramiento agrícola.

\section{Palabras clave:}

cadena lechera; extensión agrícola; asesoramiento privado; Perú

\section{Privatization of Agrarian Consultancy: Consequences for Milk Producers of the Mantaro Valley, Peru}

\begin{abstract}
Since the 1980's and the 1990's, the role of the private sector in agrarian consultancy has seen an increase worldwide. An evidence of such evolution can be seen in the Mantaro Valley (Peru), in the context of small agriculture. In order to assess its consequences, we carried out interviews with supplies providers, extension workers, and producers. In this case, private consultancy is carried out alongside the sale of supplies which, even while it stimulates the access to technical support for almost all producers, it does not take into account their overall needs. This research shows the relevance of a better coordination between the public and private actors of the agrarian consultancy system.
\end{abstract}

Keywords:

milk production chain; agrarian extension; private consultancy; Peru 


\title{
La privatisation de l'assistance agricole: conséquences pour les producteurs laitiers de la vallée Mantaro au Pérou
}

\author{
Résumé \\ Au niveau mondial, le rôle du secteur privé dans l'assistance agricole vient en augmentation depuis les \\ années quatre-vingts. Une évidence de cette évolution s'observe dans la vallée Mantaro (Pérou), dans \\ un contexte de petite agriculture. Pour évaluer les conséquences, on a réalisées des entretiens avec les \\ fournisseurs de produits, promoteurs et producteurs. Dans ce cas, l'assistance privée se réalise d'une manière \\ associée à la vente de produits, même si cette assistance aide avec l'accès de l'appui technique à presque tous \\ les producteurs, elle n'a pas en compte leurs besoins globales. Cette recherche montre l'importance d'une \\ meilleure coordination entre les acteurs publics et privées du système d'assistance agricole.
}

Mots clés:

chaîne laitier; étendue agricole; assistance privée; au Pérou

\section{Privatização do aconselhamento agrícola: consequências para os produtores leiteiros do vale de Mantaro, Peru}

\author{
Resumo \\ No nível mundial, o papel do setor privado no aconselhamento agrícola tem se acrescentado desde os \\ anos oitenta e noventa. Um sinal de tal evolução é observado no vale de Mantaro (Peru), em um contexto \\ de pequena agricultura. Para avaliar suas consequências, realizaram-se entrevistas com fornecedores de \\ insumos, extensionistas e produtores. Neste caso, o aconselhamento privado foi realizado de forma associada \\ à venda de insumos que, enquanto favorecendo o acesso de um apoio técnico a quase todos os produtores, \\ não considera suas necessidades globais. Esta pesquisa demonstra a importância de uma melhor coordenação \\ entre os atores públicos e privados do sistema de aconselhamento agrícola.
}

Palavras-chave:

cadeia leiteira; extensão agrícola; aconselhamento privado; Peru 


\section{La privatización de los servicios de asesoramiento}

El retiro del Estado se encuentra en el centro de los debates sobre las reformas de los servicios de asesoramiento agrícola en el mundo (Berdegué, 2002; Faure, Desjeux y Gasselin, 20II). Puede tomar distintas formas, como lo demuestra Rivera (2000), incluyendo: (i) una descentralización de los servicios basada en fondos públicos y manejada en el nivel regional, (ii) un otorgamiento de los servicios por parte del Estado a empresas privadas, (iii) una comercialización de los servicios por parte de las instituciones públicas con un financiamiento compartido entre el Estado y los productores, y (iv) una privatización completa.

Frente a esta diversidad de situaciones y debido a la poca eficiencia de los servicios públicos de asesoramiento agrícola, la privatización ha sido vista por la mayoría de las organizaciones internacionales como una opción de mejora. Por ejemplo, Anderson y Feder (2004) muestran que un sistema de servicios de asesoramiento se puede mejorar en los países que tienen dificultades para financiar servicios públicos, cuando el mismo está basado en una organización descentralizada y con proveedores privados.

El sector privado, incluidos los proveedores de insumos y equipos agrícolas, brinda cada vez más servicios de asesoramiento para promover sus actividades comerciales. Sin embargo, son pocos los estudios sobre la pertinencia de sus estrategias y prácticas de asesoramiento. Mirani, Bukhari y Narejo (2007), en Pakistán, o Goulet (20II), en Francia, demuestran que se pueden prestar servicios de asesoramiento de calidad invirtiendo en recursos humanos, para tener extensionistas capacitados.

Generar un sector privado amplio y eficiente requiere de un replanteamiento del papel del Estado, y de las relaciones entre los proveedores públicos y privados. Algunos autores (Anderson y Feder, 2004; Kidd, Lamers, Ficarelli y Hoffmann, 2000) consideran que el Estado tiene que seguir desempeñando un rolen las zonas más desfavorecidas y para los agricultores más pobres; otros destacan que la evolución hacia un sistema privatizado no es sencilla (Rivera y Zijp, 2002) y exige una aclaración de las funciones de cada una de las instituciones, oportunidades económicas para financiar los servicios de asesoramiento, proveedores de servicios de asesoría con capacidades adecuadas y agricultores capaces de expresar demandas claras.

Por último, la privatización de los servicios de asesoramiento implica que el Estado desarrolle nuevas funciones para regular las relaciones entre los actores y así controlar el respeto de los intereses públicos (Labarthe, 2005; Klerkx, Grip y Leeuwis, 2006). En esa línea, la intervención pública debe favorecer la evolución cualitativa del sistema de asesoramiento hacia 'redes de innovación'; es decir, hacia un sistema 
en el cual interactúen varios actores del desarrollo rural (agricultores, proveedores, extensionistas, industriales, políticos, investigadores, entre otros) para producir tanto conocimientos como aprendizajes (Dulcire, 20I4). No obstante, no todos los gobiernos disponen de las capacidades financieras y humanas necesarias -incluso la voluntad política- para hacerlo.

La privatización de los servicios de asesoramiento a veces se entiende como un medio para transferir el gasto público a los beneficiarios finales. Algunos sistemas privados, con relaciones comerciales entre clientes y proveedores, han demostrado su eficacia en el caso de la agricultura intensiva en los países industrializados (Kidd et al., 2000). Pese a ello, y en general, se admite que la mayoría de los agricultores -y no solo los de los países en desarrollo- no puede asumir por sí misma el costo de tales servicios (Klerkx et al., 2009).

$\mathrm{Al}$ admitir el papel de la extensión privada y las dificultades de los productores para financiarlo, el debate sobre este tema se centra en las posibles alternativas de financiación. De hecho, es posible combinar la prestación de un servicio de asesoramiento de un operador privado y/o público con la financiación de fondos públicos y/o privados (Birner, Davis, Pender, Nkonya, Anandajayasekeram, Ekboir... Cohen, 2009). Tal alianza público-privada (Christoplos, 2010) puede representar una oportunidad que da más flexibilidad al sistema de servicios de asesoramiento.

A pesar de ello, diferentes estudios también mencionan varios riesgos de la privatización (Kidd et al., 2000; Labarthe, 2005; Klerkx et al., 2006), como la difusión limitada de las innovaciones complejas; las pocas preocupaciones por los temas ambientales o de la complejidad del sistema de producción; la fragmentación de los temas de asesoramiento para mejorar las posibilidades de comercialización de los servicios; la preferencia de una transferencia de tecnología con poca capacitación de los productores; la reducción de los intercambios de información entre agricultores, que no quieren compartir conocimientos comprados, y la exclusión que genera la selección de los agricultores capaces de comprar servicios de asesoría. Para limitar estos riesgos en algunos países de América Latina, volvió a fortalecerse a mediados de la primera década del siglo XXI la extensión agrícola pública (Aguirre, 20I2), pero ese no fue el caso en otros países como el Perú (id.). Sin embargo, los efectos del abandono progresivo por parte del Estado de los servicios de asesoramiento agrícola y el aumento de servicios privados, aún no han sido suficientemente estudiados en países del Sur. 
La investigación llevada a cabo por la Universidad de la Molina, en Lima, y el Centro de Cooperación Internacional de Investigación Agronómica para el Desarrollo (CIRAD) tenía como propósito el fortalecimiento de los pequeños productores lecheros en la zona andina del valle del Mantaro en Perú (2010 a 20I2), y aporta elementos de respuesta para entender mejor la situación de los servicios de extensión.

La zona geográfica es relevante para este estudio, porque la mayoría de los productores es pequeña y allí el sector privado ha tomado cada vez más peso en el sistema de asesoramiento, con base en la combinación de asesoramiento y venta de insumos.

El objetivo del artículo es analizar a partir de este estudio de caso la evolución del sistema de asesoramiento agrícola, y sus consecuencias en la cobertura del servicio, la adaptación de los contenidos de la asistencia, su financiamiento y las formas de coordinación entre actores. Los resultados de la investigación pueden ser de utilidad para los actores locales, con el fin de mejorar el sistema, pero también para investigaciones en otras zonas, que permitan establecer comparaciones y una evaluación de los efectos de la privatización en los servicios de extensión agrícola para los pequeños productores.

\section{Metodología del estudio}

\section{Elección de la zona de estudio}

La pequeña agricultura del valle del Mantaro $(3200 \mathrm{msnm})$ se caracteriza por poseer una ganadería lechera con diferentes cadenas organizadas y tres formas de procesamiento: artesanal y familiar, de pequeña empresa, e industrial. Existe una diferenciación entre las explotaciones lecheras (Cortijo, Faure y Le Gal, 20ro): las pequeñas tienen menos de tres vacas; las medianas, entre cuatro y diez vacas, y las más grandes, más de diez y hasta cien.

La leche es un producto estratégico para los pequeños productores, por la diversidad de fuentes de comercialización a buen precio y por la estabilidad del mercado. Estos sistemas ganaderos están basados en parcelas forrajeras con riego y vacas en establo. En pro de aumentar la producción lechera del hato, los productores compran insumos para el manejo de sus pastos (semillas, fertilizantes) y del ganado (concentrado, productos veterinarios). La provincia de Concepción, una de las nueve que conforman el departamento'de Junín, fue elegida para esta investigación por ser la zona de mayor

I Un departamento es una entidad subnacional mayor del Perú, administrado por un gobierno regional.

Cada departamento es dividido en provincias, que son subdivididas en distritos. 
producción lechera del valle. Este mismo departamento cuenta con 4500 explotaciones ganaderas y la provincia de Concepción, con I300, de manera que produce el 30\% de la leche del departamento (Dirección General de Agricultura de Junín, 20II).

\section{Entrevistas a actores de los servicios de extensión}

Para este estudio se planteó caracterizar y comparar los distintos servicios de extensión, tanto privados como públicos, que se ofrecen a los productores lecheros; esto, por la necesidad de comprender mejor estas actividades en esa provincia. En una primera etapa se identificaron los distintos proveedores de servicios de extensión (instituciones públicas, ONG, empresas lecheras, casas comerciales proveedoras de insumos) que intervienen en la provincia de Concepción. Posteriormente se realizaron treinta y cinco entrevistas semiestructuradas (que abarca preguntas cerradas y abiertas) con cada responsable de los proveedores y con uno o más de sus extensionistas, con la finalidad de caracterizar la historia del proveedor, su zona de intervención, los temas objetos del servicio, las actividades desarrolladas, los mecanismos de financiamiento, las relaciones con los productores y otros actores, y las percepciones de los proveedores sobre el servicio.

En una segunda etapa se construyó una muestra basada en un total de cuarenta productores de leche, teniendo en cuenta que la demanda de servicios puede ser variable con base en el tamaño de hato (menos de tres vacas, entre cuatro y diez vacas, entre once y veinte, entre veintiuno y treinta, más de treinta vacas). Con ellos se realizaron entrevistas para caracterizar su sistema de producción, incluso su consumo de insumos, los apoyos de parte de técnicos públicos o privados, y su evolución en los diez últimos años en los temas objetos del servicio, las actividades desarrolladas, la calidad de la relación con los técnicos, el pago de la asesoría y sus percepciones sobre los servicios.

Finalmente, estos datos fueron procesados para un análisis de la situación actual de los servicios de asesoramiento del valle del Mantaro, en cuanto a los servicios recibidos por los productores según el tamaño del hato, los servicios brindados según el tipo de proveedor de servicios, la calidad de estos, el costo y financiamiento de los servicios, y las relaciones entre proveedores de servicios y mecanismos de coordinación. Parte de los resultados de este estudio fue presentada en un informe (Huamanyauri, 2013).

También se dio valor a datos de otros estudios llevados a cabo con la participación de unos de los autores de este artículo, para confirmar los resultados: caracterización de los sistemas de producción (Laporte, Faure y Le Gal, 2008), análisis de las explotaciones ganaderas y sus relaciones con las procesadoras (Cortijo et al., 2010), y análisis de la cadena de leche en el valle del Mantaro (Gamboa, 20I2). Por último, 
se realizó un taller a finales del año 2012 con varios actores de las cadenas lecheras del valle (productores, técnicos, responsables de proveedores de servicios, empresas lecheras, casas comerciales), que incluyó la presentación de los resultados, discusiones y trabajos por grupo. Este ejercicio sirvió para compartir, validar y afinar los resultados. También, para construir con los actores recomendaciones para mejorar el sistema de asesoramiento en el valle.

\section{La privatización de los servicios de asesoramiento}

El sistema público de extensión agraria surgió en el Perú en el año 1942 y fue reformado a finales de la década de los ochenta, lo que brindó oportunidades a nuevos actores, hasta a las empresas privadas, de incrementar sus ofertas. En el valle del Mantaro, el sistema de asesoramiento ha evolucionado más rápidamente desde 2005, cuando el Estado peruano disminuyó los recursos de las agencias agrarias ${ }^{2}$ y transfirió el mandato del asesoramiento a los gobiernos regionales.

Al mismo tiempo, el mercado de los insumos se fortaleció con la implantación de varias firmas comerciales en el valle que promueven sus productos con vendedores y técnicos, y las empresas de procesamiento lácteo también empezaron a brindar asesoría a sus proveedores de leche. Por otra parte, las ONG abandonaron la provincia de Concepción para actuar en zonas más altas y desfavorecidas. Los párrafos siguientes analizan con más detalles este proceso.

\section{El retiro progresivo del Estado}

La agencia agraria ha jugado un papel histórico en la difusión de tecnologías enfocadas al aumento de la productividad y de la producción agrícola. Su función se ha transformado, frente a las reducciones presupuestarias, en el marco de la descentralización, por lo que actualmente intenta, sin mucha experiencia, cumplir un rol de coordinación entre los distintos actores e instituciones, apoyando de esta manera el desarrollo rural y reforzando las cadenas agroproductoras.

En nombre del Ministerio de Agricultura, Gutiérrez (2007) propone otra opción, que comprendeuna redefinición del rol de las agencias agrarias en Perú para fortalecer

2 Una agencia agraria es una entidad del Ministerio de Agricultura ubicada en los departamentos para proveer servicios de asesoramiento agrícola. 
las capacidades de los gobiernos regionales y municipales en temas de gestión y desarrollo del territorio rural.

Otras organizaciones públicas y universidades difunden informaciones agrícolas en el valle del Mantaro, lo que contribuye a desarrollar un sistema local de innovación. Tres institutos de investigación públicos (el Instituto Nacional de Innovación Agraria, Inia; el Servicio Nacional de Sanidad Agraria, Senasa; el Instituto Veterinario de Investigaciones Tropicales y de Altura, Ivita) y las dos universidades locales (Universidad Nacional del Centro del Perú, pública, y la Universidad Peruana de los Andes, privada) desarrollan, paralelamente a sus actividades principales y a través de capacitaciones en aula, prácticas de apoyo a los productores con el fin de difundir conocimientos científicos de mejoramiento genético, sanidad animal, alimentación, manejo de pastos con semillas mejoradas, entre otros.

Para estas actividades, movilizan a sus técnicos, o estudiantes en el caso de las universidades, o contratan a terceros de vez en cuando. Por su parte, el Programa Sectorial de Irrigación ofrece apoyo para fortalecer las asociaciones de riego. En este contexto, se puede decir que el Estado sigue presente pero de manera limitada.

El punto más importante es que la descentralización ha favorecido el desarrollo de proyectos públicos financiados por el Gobierno Regional de Junín. No obstante, el carácter de corto plazo de este tipo de proyectos contribuye a la contratación puntual de extensionistas que poseen una experiencia limitada, ya que se contrata a nuevos extensionistas cada vez que se inicia un nuevo proyecto. Entre ellos, el proyecto Progale (Programa de Mejoramiento Genético y de Asistencia Técnica para la Ganadería Lechera), para el fortalecimiento de la actividad ganadera, contrató a once técnicos que trabajaron en 2012 con 250 productores procedentes de varias provincias del departamento, incluyendo Concepción ${ }^{3}$. Eso se traduce en un porcentaje débil (6\%) de productores del departamento atendidos por los técnicos de Progale.

Los objetivos de estos proyectos siguen siendo los mismos que los promovidos antes por la agencia agraria; esto es, todavía enfocados en temas técnicos para aumentar la producción y la productividad. Se tocan primero los temas de sanidad animal y mejoramiento genético, y luego, los temas de manejo de pastos, alimentación de los animales y manejo del ganado (infraestructura, higiene). Por eso se movilizan principalmente dos herramientas: (i) los talleres de capacitación, tipo charlas, y (ii) la

3 Un productor puede acceder a los servicios de los proyectos a través de las organizaciones de productores, que funcionan como intermediarias y no como proveedoras de servicios a sus socios. 
asistencia técnica individual estructurada por un programa de intervención elaborado cada semana, con base en llamadas telefónicas de los productores.

La participación del productor está motivada por el acceso a servicios individuales (asistencia técnica, inseminación, compra de medicamentos a precios cómodos y al contado, entre otros), ya que los productores expresan poco interés en las charlas. A su parecer, son muy académicas, es decir no tienen relación directa con sus condiciones y prácticas agrícolas.

\section{El auge del sector privado}

En este contexto de retiro del Estado, varios tipos de actores privados han aprovechado la disminución de la extensión pública para proponer, por razones distintas, su asistencia técnica a los productores. Los actores privados pueden ser empresas privadas, $\mathrm{ONG}$ u organizaciones de productores.

En el valle del Mantaro, las ONG actualmente centran su trabajo en las zonas altas, donde el nivel de pobreza atrae más financiamientos internacionales ${ }^{4}$. No existen tampoco sólidas organizaciones de productores en la zona y las pocas que hay fueron creadas por el Ministerio de Agricultura para facilitar las relaciones entre los productores y los extensionistas. Lo más importante y visible en el valle es el desarrollo de las actividades privadas a través de casas comerciales y técnicos particulares.

\section{El papel creciente de las casas comerciales}

Las casas comerciales que venden medicamentos, concentrados y semillas de pastos, proveen informaciones sobre su uso cuando el productor los compra. Las casas comerciales más grandes en términos de volumen de venta (Mateos, Hortus, Eoch, Agroveterinaria, Agrorural, Fertisol, Pfizer, Química Suiza y otras) empezaron a instalarse en la zona a partir del 2000, con una sede en la ciudad principal del valle (Huancayo) y muchas veces con tiendas en los pueblos.

La calidad de la información brindada al productor depende del nivel de formación de los vendedores, y de sus posibilidades para ofrecer informaciones y capacitaciones adicionales a través de convenios con universidades o transnacionales que venden los productos. De las siete casas comerciales, cinco ofrecen técnicos que

\footnotetext{
4 La única ONG que trabaja ahora el tema de la ganadería en el valle atiende a 150 productoras, pero fuera
} de la provincia de Concepción. 
van al campo a dar charlas y a brindar asistencia técnica, lo que facilita un servicio de calidad. Por ejemplo, la casa comercial Fertisol ocupa tres personas en tarea de venta que realizan visitas de campo en el distrito de Concepción; atendieron en 2012 a 500 productores (38\% de los productores de la provincia). Existe una competencia entre las casas comerciales, y la calidad del asesoramiento es un medio para atraer y fidelizar clientes.

El surgimiento de los particulares

En la zona de estudio también se observa la presencia de técnicos independientes que no trabajan con el sector público ni para una comercializadora. Se identifican cuatro categorías: veterinarios, ingenieros zootecnistas o agrónomos, técnicos agropecuarios y otros (estudiantes y productores). La última categoría tiene la denominación de 'técnicos empíricos', que aprendieron observando y trabajando. Todos ellos tienen sus propios clientes y ejercen otra actividad (docencia, intervención puntual en proyectos, producción). En el Cuadro i se observa que los veterinarios e ingenieros son, en mayor porcentaje, especialistas en inseminación artificial y reproducción, así comolos técnicos agropecuarios en sanidad animal.

Cuadro 1. Actividades de los proveedores particulares de acuerdo con su profesión (\%)

\begin{tabular}{lcccc}
\hline Profesión Actividad & $\begin{array}{c}\text { Veterinario } \\
(\mathrm{N}=5)\end{array}$ & $\begin{array}{c}\text { Ingeniero } \\
(\mathrm{N}=5)\end{array}$ & $\begin{array}{c}\text { Técnico } \\
(\mathrm{N}=5)\end{array}$ & $\begin{array}{c}\text { Otro } \\
(\mathrm{N}=1)\end{array}$ \\
\hline Inseminación artificial y reproducción & 80 & 60 & 40 & 0 \\
\hline Sanidad animal & 40 & 0 & 80 & I00 \\
\hline Manejo del ganado & 20 & 40 & 20 & 0 \\
\hline Manejo de pastos y forrajes & 0 & 20 & 0 & \\
\hline
\end{tabular}

Fuente: entrevistas de 2011 a 2012, elaboración propia 
Todos estos proveedores venden insumos para cubrir sus costos y tener ingresos, lo que aumenta la competición entre vendedores de insumos, inclusive las casas comerciales. Proveen información sobre el uso de los insumos y brindan asesoramiento en su campo de especialidad. Los productores acuden a estos servicios porque valoran la proximidad, tienen confianza, piensan que la información brindada es de calidad, o estiman que los precios o condiciones de venta de los insumos son más interesantes que lo que pueden conseguir con las casas comerciales. Algunos de estos proveedores tienen una oferta de servicios bien definida.

Por ejemplo, un veterinario de Concepción cuenta con I20 clientes registrados ( $9 \%$ de los ganaderos de la provincia), tiene una relación más estrecha con productores que crían entre 20 y 30 vacas, y posee un programa de visitas regulares con 50 de ellos (dos visitas por mes por productor), asesorando a otros clientes a partir de llamadas telefónicas. Sus intervenciones más importantes son: sanidad animal (casos agudos y venta de medicamentos) e inseminación. Con algunos productores se lleva a cabo un programa de intervención que incluye aspectos ligados a la sanidad animal, al mejoramiento genético, así como al manejo del ganado y de los costos de producción. El veterinario cubre sus costos a través de la venta de medicamentos, por intervención especial (inseminación, actos de cirugía), o por cuota mensual en el caso de un programa de intervención (de 50 a 150 soles $^{5}$ por mes según el tamaño del hato).

\section{Las intervenciones tímidas de las empresas lecheras}

Algunas empresas lecheras suelen invertir en la extensión para garantizar una producción lechera regular y de calidad con clientes fieles. En el valle del Mantaro son de dos tipos las que tienen capacidades para desarrollar estas actividades (Laporte et al., 2008; Cortijo et al., 2010): las empresas lecheras Gloria o Nestlé, que intervienen en todo el Perú, y las procesadoras familiares de tamaño medio (2000 a 3000 litros/día).

La lechera Gloria es la única del valle que tiene un técnico de tiempo completo dedicado a asistir a los productores. Organiza charlas en varias comunidades con diferentes temas y provee asistencia técnica individual. Atendió en 2012 a alrededor de 400 productores en el departamento, incluyendo la provincia de Concepción (9\% de los ganaderos del departamento). Como en el caso de Progale, los productores valoran más la asistencia técnica individual y menos las charlas, aunque las de Gloria suelen ser más relacionadas con las prácticas de los productores.

5 Un sol (S) = USo,35 en 2013 
Las procesadoras familiares no tienen recursos para contratar técnicos que apoyen a los productores, pero desarrollan actividades para asegurar sus abastecimientos en leche. Por ejemplo, una de ellas contrata veterinarios para dar capacitaciones y financia parte del costo de este servicio. Aprovechando la presencia de un agrónomo en la familia que trabaja en la empresa, otra lechería familiar organiza charlas para capacitar a los ganaderos y disemina informaciones sobre las semillas de pasto. Una tercera consiguió el financiamiento (2008 a 20ro) de un proyecto internacional para contratar a un extensionista que ayudara en la implementación de una organización de productores, así como en su capacitación.

Según los productores entrevistados, este tipo de apoyo es valioso pero no toma en cuenta lo suficiente el contexto de explotaciones más pequeñas, por ejemplo: ¿cómo mejorar la higiene cuando uno no tiene acceso al agua potable? Además, para ellos este tipo de apoyo no reemplaza la necesidad de una asistencia técnica individual.

Como resultado general, las intervenciones de las procesadoras en el sistema de asesoramiento se pueden apreciar, pero la inversión es aún limitada. Exceptuando el caso de Gloria, el número de productores atendidos por las empresas no es significativo (alrededor de roo en la provincia de Concepción, o7\% de los ganaderos) y las actividades de extensión no suelen ser regulares.

\section{Consecuencias de la privatización parcial de la extensión}

La privatización parcial de la extensión tiene impactos significativos. Se tiene una mayor cantidad de productores atendidos por la mayor presencia de proveedores de servicios y por la competición entre ellos, aunque todavía en un marco únicamente técnico, siendo una actividad financiada por la venta de insumos.

\section{Una cobertura más amplia de los productores}

Esta privatización parcial de la extensión en el valle llama la atención pues no se ha traducido como una forma de exclusión de los productores, tal como lo menciona la literatura (Cristovao, Koutsouris y Kügler, 20I2), por lo menos en la zona de estudio, que es la más productiva y moderna del departamento. Si bien Fernández-Baca y Bojorquez (1994) indicaron que en 1990 alrededor de la mitad de los encuestados recurría a un técnico particular cuando sus animales se enfermaban, ahora la realidad es diferente y casi todos los productores (93\% de los entrevistados) tiene acceso a asistencia técnica personalizada, es decir en su propio establo. Dicha asistencia técnica es brindada en 
su mayoría por las casas comerciales o los particulares, y solo por parte de técnicos de Progale o Gloria. La asistencia es gratuita, lo que explica en gran parte este éxito.

Además, el 38\% de los productores ha participado en capacitaciones en aulas organizadas por las instituciones públicas, el proyecto Progale o la empresa Gloria. Estas cifras son confirmadas por el estudio de Gamboa (20I2): 94\% de los productores entrevistados tiene acceso a asistencia técnica y $29 \%$ a capacitaciones.

Tal intensidad de asistencia técnica está ligada al auge del sector privado, pero también al desarrollo del teléfono inalámbrico. Así, el 73\% de los productores llama por celular cuando requiere de un apoyo y, generalmente, cuando los animales tienen problemas de sanidad. En cuanto a los técnicos de Progale, estos organizan sus programas de visitas llamando o contestando llamadas por teléfono. Con respecto al técnico de la empresa Gloria, sus visitas son de tres tipos: programadas con anticipación por la empresa, con Koutsouris, A. y Kügler en una demanda de un productor y según un problema de calidad de la leche entregada.

Hay que destacar que tanto las instituciones públicas -Progale- como privadasGloria-concentran sus esfuerzos con los productores más grandes, porque tienen más potencialidad de producción (véase el Cuadro 2), lo que genera una forma de desigualdad en el acceso al asesoramiento. Por otro lado, las casas comerciales y los particulares trabajan con todos los productores, abarcan temas ligados al uso de insumos y financian sus servicios a través de la venta de los mismos. Tal sistema se traduce en una atención a todos los productores, en la cual el uso de insumos es el primer propósito de la intervención de los técnicos. Sin embargo, ya se ha destacado que el número de productores atendidos por técnico es muy variable (entre 25 por los técnicos de Progale y 400 por el técnico de Gloria).

Cuadro 2. Porcentaje de productores de leche, por clase de rebaño, que reciben capacitaciones y asistencia técnica, por tipo de estructura

\begin{tabular}{lccccc}
\hline \multicolumn{1}{c}{ Número de vacunos } & $\begin{array}{c}1 \mathrm{~A} 3 \\
(\mathrm{~N}=12)\end{array}$ & $\begin{array}{c}4 \mathrm{~A} 10 \\
(\mathrm{~N}=12)\end{array}$ & $\begin{array}{c}11 \mathrm{~A} 20 \\
(\mathrm{~N}=10)\end{array}$ & $\begin{array}{c}21 \mathrm{~A} 30 \\
(\mathrm{~N}=4)\end{array}$ & $\begin{array}{c}>30 \\
(\mathrm{~N}=4)\end{array}$ \\
\hline $\begin{array}{l}\text { Capacitaciones de diversas } \\
\text { instituciones públicas }\end{array}$ & 0 & 33 & 60 & 50 & 50 \\
\hline $\begin{array}{l}\text { Asistencia técnica de } \\
\begin{array}{l}\text { Progale y Gloria } \\
\text { Asistencia técnica de particulares }\end{array}\end{array}$ & 100 & 83 & 100 & 100 & 50 \\
\hline $\begin{array}{l}\text { Asistencia técnica de las } \\
\text { casas comerciales }\end{array}$ & 100 & 92 & 90 & 100 & 100 \\
\hline
\end{tabular}

Fuente: entrevistas de 201 a 20I2, elaboración propia 


\section{Una asistencia aún más técnica}

La práctica extensionista se ha enfocado en temas únicamente técnicos, basados en los componentes y principios de la revolución verde (mejoramiento genético, sanidad animal, alimentación, intensificación de los pastos, entre otros), y manejados de manera separada, lo que Landini (20I2) califica como "persistencia del difusionismo" a nivel de América Latina.

Las instituciones públicas, así como las privadas, siguen manejando este enfoque "tecnicista" para sus capacitaciones, sin adaptarlas a la diversidad de las explotaciones, y de las capacidades y necesidades de las familias (véase la figura I), es decir sin proponer modelos alternativos tanto por falta de tiempo (número de familias atendidas por técnico) como por falta de formación en este área.

Esta característica es de mayor prominencia en el caso del sector privado, en el cual los temas más tratados son el fortalecimiento del ganado por uso de vitaminas, el control de los parásitos y de las mastitis por uso de medicinas, la inseminación artificial, y la implantación de pastos con semillas mejoradas. La privatización parcial de la extensión ha orientado la oferta de conocimientos y nuevas tecnologías, aprovechándose de una interacción positiva entre: (i) la oferta concentrada en estos temas, los cuales promueven el uso de insumos químicos, alimentos balanceados y medicamentos y (ii) las demandas de los productores para una extensión especializada, que produzca impactos rápidos y cuantificables en la solución de casos urgentes.

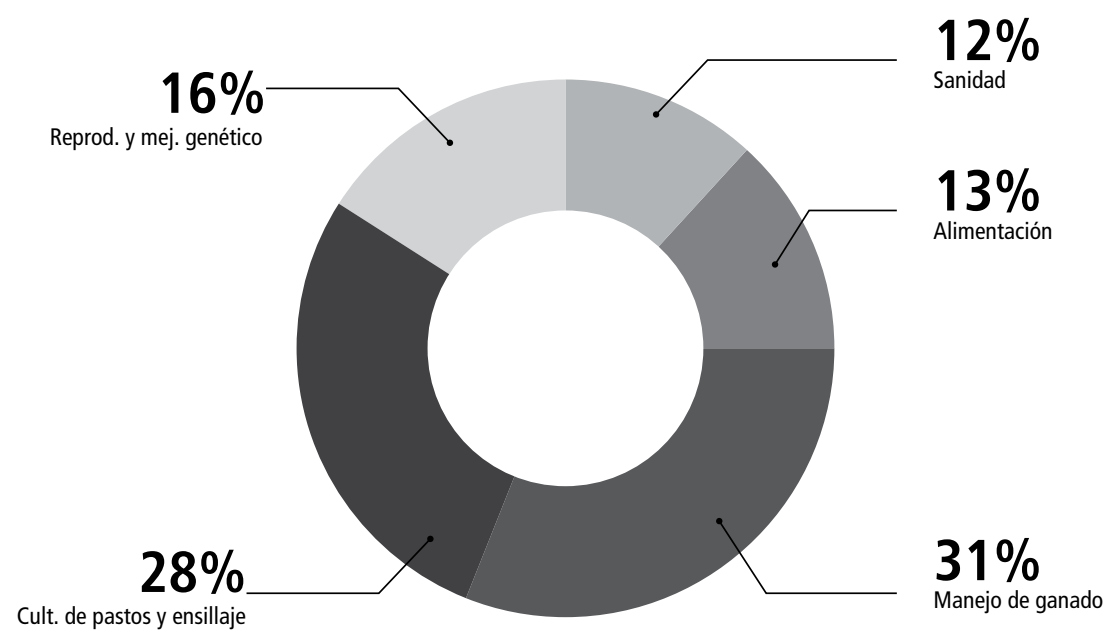

Figura i. Distribución de los temas tecnicistas de capacitación (\%)

Fuente: entrevistas de $201 \mathrm{I}$ a 20I2, elaboración propia 
Es decir, los servicios de asesoramiento no consideran como importantes unos temas que solicitan los productores; por ejemplo, el manejo de las relaciones entre las diversas actividades agrícolas y ganaderas de la finca, ni el manejo adecuado de las parcelas de forraje con base en la evolución de la demanda de alimentos del hato a lo largo del año, ni el mejoramiento de los resultados económicos y financieros de la explotación, ni la seguridad alimentaria de la familia (Bienz y Le Gal, 2012).

De los tres principales componentes para la extensión identificados por Röling y Groot (1998): transferencia de tecnología, asesoramiento y apoyo a procesos de aprendizaje, el único que se aplica en el valle del Mantaro es el primero. Tal enfoque tiene limitaciones, pues no facilita los aprendizajes de los productores, no considera todas sus necesidades y tampoco las capacidades innovadoras de los actores locales (Scoones y Thompson, 2009).

\section{Un financiamiento asegurado por la venta de insumos}

Si bien el servicio de extensión brindado por las instituciones públicas es gratuito y financiado completamente por el sector público, se aprecia una fuerte disminución del financiamiento directo por parte del Estado, tal como lo demuestra la situación de la Agencia Agraria. Esto ha fomentado el desarrollo de proyectos financiados por el gobierno regional, entre los cuales está el actual Progale, que invierte en la extensión pero con altibajos que generan una falta de continuidad en sus actividades. Con el proyecto financiado por el Banco Mundial (2008 a 2010) Innovación y Competitividad para el Agro Peruano (Incagro), se experimentó un mecanismo novedoso de fondos competitivos para el financiamiento de la investigación y la extensión agrícola, como respuesta a demandas de los actores locales. Tales fondos competitivos son comunes en muchos países para facilitar la diversificación de la oferta de servicios de asesoramiento (Faure et al., 20II). Sin embargo, Incagro financió solamente un proyecto en el valle del Mantaro 6 , y el Estado no continuó con dicho mecanismo cuando terminó el proyecto del Banco Mundial.

En el caso de los servicios de extensión privada, estos son financiados por la venta de insumos (medicamentos, alimentos balanceados, semillas, fertilizantes) o por la compra de leche (el caso de Gloria y otras empresas que brindan servicios). Dicho financiamiento, ligado a la venta de insumos, orienta los consejos del proveedor

6 Una procesadora de leche fue financiadacon este mecanismo en el valle, lo que facilitó la implementación de una organización de productores para agilizar las relaciones entre ellos y los procesadores. 
en función de sus productos ofrecidos, con el fin de que el productor compre más insumos: tal como lo dice uno de ellos, "todo se resuelve con vitaminas e inyección".

No obstante, para asegurar la venta de insumos, y más que todo para que los productores continúen siendo fieles, el proveedor debe asegurarse de brindar un servicio de calidad que se evalúe en función de la resolución del problema enunciado por el productor. Solo algunos productores ( $7 \%$ según el estudio) pagan de contado a los particulares para tener acceso a una asistencia técnica con visitas regulares en la finca, sin tener que comprar sistemáticamente insumos. Para muchos particulares, tal nivel de actividad no es económicamente suficiente y tienen que desarrollar otras actividades (consultoría, producción agrícola y otros).

Cortijo et al. (20ro) calcularon que los costos de forraje y de concentrados comprados, así como de las medidas para asegurar la sanidad y la reproducción, representan un $38 \%$ de los costos de producción de las explotaciones lecheras y un $50 \%$ si se incluyen los fertilizantes para los pastos. Estos últimos costos representan entre 1000 y 1400 soles/vaca. El estudio de Gamboa (2012) concluye valores semejantes. Estas cifras demuestran la importancia del mercado de los insumos, tomando en cuenta los 4500 ganaderos del valle del Mantaro, es decir entre 15 y 20 millones de soles.

\section{Una fuerte competencia entre extensionistas que requieren capacitaciones}

La privatización parcial de la extensión ha generado competencia entre los proveedores de servicios, lo que ocurre en otros países (Labarthe, 2005). Pero la oferta del sector privado y del sector público no es tan distinta, ni en los temas ni en cuanto a la metodología de extensión. Los productores pueden aprovechar tal situación para seleccionar la oferta que más les conviene. El caso de la inseminación es un buen ejemplo de esta competencia, porque los veterinarios, los inseminadores privados o los inseminadores de la institución pública proponen el mismo servicio con precios muy variables: de 15 a 600 soles/vaca.

Pese a ello, esta competencia no garantiza que los proveedores brinden la información adecuada y completa para que los productores puedan tomar una decisión óptima. Tomando de nuevo el ejemplo de la inseminación, son muy pocos los productores que tienen acceso a un servicio de calidad para elegir una raza y la inseminación adecuada con base en una estrategia de mejoramiento del ganado. Además, es preocupante la dura competencia que pueden enfrentar los extensionistas con diploma cuando compiten con "extensionistas empíricos", que no tienen una 
formación adecuada y venden servicios a precios baratos para atraer a clientes, aunque sin garantía de la calidad de su servicio.

Debido a que los extensionistas necesitan acceder a informaciones nuevas para mantener sus competencias, una capacitación constante resulta indispensable, pero no hay un sistema de formación apropiado. Las universidades ofrecen ocasionalmente cursos con temas relacionados a la producción, a los cuales son muchos los extensionistas que solicitan participar. Empero, no existen cursos para mejorar su forma de asesorar: organizar un taller, fomentar las interacciones, generar conocimientos al interactuar entre saberes locales y académicos, por ejemplo.

$\mathrm{Al}$ tener dificultades, unos contactan a colegas de su red personal para conseguir informaciones y respaldo, y otros organizan reuniones “informales" entre ellos mismos para compartir experiencias y saber-hacer. A pesar de ello, las mayores fuentes de información (afiches, documentos y otros) y de capacitación (charlas o intercambios individuales), tanto de los extensionistas como de los veterinarios, son las empresas privadas de insumos, que abastecen las casas comerciales del valle. Tal tendencia no garantiza una información imparcial y aumenta aún más el enfoque de la extensión hacia la promoción y venta de insumos.

\section{Una falta de coordinación entre actores locales e instituciones públicas}

Si tomamos la clasificación de Rivera (2000), el Estado ha elegido una forma de retiro de la extensión, fundado en dos ejes: la privatización y la descentralización. La primera genera competencia entre proveedores privados, lo que puede resultar positivo para los productores, y entre proveedores privados y públicos porque se enfocan en los mismos temas; aun así, esto último resulta más bien en un desgaste de recursos.

La descentralización, por otro lado, no ha logrado fomentar nuevos servicios de asesoramiento importantes sino que ha favorecido una falta de coordinación entre los proveedores de servicios, puesto que las agencias agrarias no han podido cumplir con este rol. En algunos países, las organizaciones de productores juegan un papel importante en la coordinación de la oferta de servicios (Le Coq, Faure y Sáenz, 20ı。) pero este no es el caso en el valle del Mantaro por las debilidades que se enfrentan. Sin embargo, una mejor coordinación entre actores locales es necesaria para fomentar un desarrollo rural sostenible (Puente, Gallego y Vidueira, 20II). Tal observación la hicieron los extensionistas durante el último taller del año 20I2, por las tensiones que observaban en su momento entre proveedores de servicios. 


\section{Recomendaciones y conclusión}

El retiro del Estado, a partir de la descentralización y de la privatización parcial de la extensión agrícola, se traduce en el caso del valle del Mantaro en el auge de las casas comerciales y de los extensionistas particulares. En consecuencia, la venta de insumos es la que financia las actividades extensionistas y orienta sus temas a componentes técnicos aislados, lo que al final aumenta los costos de producción de los productores.

No obstante, al contrario de lo enunciado en la literatura científica al analizar los efectos de la privatización para los pequeños productores (Cristovao et al., 20I2), en el caso del valle esta tendencia no los excluye por estar inmersos en una zona con importante potencialidad económica. Ahora bien, cada uno tiene algún margen de acceso a un técnico, pero sin que este se ajuste al asesoramiento de necesidades reales de los productores.

Esta conclusión puede ser consistente con la argumentación de que la privatización tiende a excluir del servicio de extensión a los productores, en los contextos en los cuales no pueden pagar por el servicio (sea directa o indirectamente, como en este caso). En este sentido, el estudio aporta estos elementos nuevos para el análisis de la privatización de los sistemas de extensión, dándole más visibilidad a un proceso poco conocido por los tomadores de decisión a nivel nacional.

El estudio permite formular cinco recomendaciones. Primero, un diálogo entre los ganaderos y sus organizaciones, por un lado, y de las instituciones, por el otro. Ello es necesario para definir conjuntamente un programa de extensión que se base en las necesidades expresadas y que tome en cuenta los recursos humanos de la zona. Tal diálogo puede facilitar el surgimiento de una coordinación entre los proveedores de servicios, por ejemplo, con la puesta en marcha de una plataforma de intercambios entre actores, que podría ser más eficiente que la creación de un ente coordinador de los actores del desarrollo rural por el posible riesgo de ser burocrático.

La segunda recomendación que nace es que el servicio público de asesoramiento tendría que desarrollar, por un lado, actividades en áreas que no compitan con el sector privado, como el manejo integral de la explotación, la gestión económica, los impactos ambientales, la práctica del riego, el acceso al mercado y demás, y por otro lado, actividades de coordinación sistémica de los actores del sector agropecuario. Lo anterior implica que tal extensión requiere de agentes públicos estables y con un nuevo perfil: que sean capaces de gestionar un enfoque sistémico con una visión amplia del desarrollo y que acompañen procesos interactivos de facilitación, no limitándose a transferir tecnologías únicamente. 
La tercera es que se hace necesario apoyar el sector privado para fortalecer las capacidades de sus extensionistas, con acceso a capacitaciones independientes de las transnacionales vendiendo insumos. Además, es relevante implementar procesos evaluativos de la calidad de su actividad para limitar la competencia con proveedores no calificados. El control de la calidad de los insumos vendidos debe ser también mejorado, reforzando y aplicando las leyes nacionales.

La cuarta recomendación está relacionada con que el fortalecimiento de las organizaciones de productores aparece como una necesidad para desarrollar una cadena de leche con relaciones eficientes entre productores y procesadoras (Vásquez y Aguilar, 20I0). Tales aprendizajes organizacionales innovadores les dan a las organizaciones de productores la capacidad de jugar un rol activo en la extensión, negociando con los sectores privado y público los apoyos requeridos por sus socios, empleando técnicos para apoyar a los productores, y/o promoviendo una forma de extensión de "campesino a campesino".

La quinta es que el auge del sector privado necesita que el Estado se posicione mejor con el fin de armar un programa de capacitación y certificación de los extensionistas tanto públicos como privados; también exige implementar un sistema de información abierto para los productores y sus organizaciones sobre el uso de los insumos químicos y la calidad de los proveedores de servicios; además, en ese sentido, es menester experimentar nuevos financiamientos de la extensión con recursos mixtos públicos y privados o con énfasis en temas que no son de interés para el sector privado.

De manera concluyente, la privatización de la extensión a través del auge de la venta de insumos puede ser una oportunidad para los pequeños productores, si el Estado es capaz de implementar mecanismos de control, de fomentar servicios de asesoramiento más sistémicos y complementarios a los del sector privado, y de fortalecer la capacidad de las organizaciones de productores para defender los intereses de sus socios. De lo contrario, la privatización de esta extensión podría favorecer un consumo excesivo de insumos, aumentando los riesgos económicos de los pequeños productores, así como los riesgos ambientales.

\section{Agradecimientos}

Los autores agradecen al Banco Mundial, que financió entre 2008 y 2012 varios proyectos sobre los sistemas agrarios y la cadena lechera en el valle Mantaro de Perú. Uno de estos proyectos incluía un estudio del sistema de asesoramiento. El autor principal participó en todos estos proyectos. 


\section{Referencias}

Aguirre, F. (2012). El nuevo impulso de la extensión rural en América Latina. Situación actual y perspectivas. Santiago de Chile: RELASER.

Anderson, J. R. y Feder G. (2004). Agricultural extension: Good intentions and hard realities. World Bank Research Observer, 19, 4I-60.

Berdegué, J. (2002). Las reformas de los sistemas de extensión en América Latina a partir de la década de los 8o. Santiago de Chile: RIMISP.

Bienz, N. y Le Gal P. Y. (20I2). Cultivating Prospective Thinking: A Gateway into the Future for Peruvian Dairy Farmers in the Mantaro Valley.Experimenting a Support Approach Based on the Use of Modelling Tools. Montpellier: CIRAD.

Birner, R., Davis, K. E., Pender, J., Nkonya, E., Anandajayasekeram, P., Ekboir, J.... Cohen, M. (2009). From best practice to best fit: a framework for designing and analyzing pluralistic agricultural advisory services worldwide. Journal of AgriculturalEducation and Extension, 15(4), 341-55.

Christoplos, I. (2010). ¿Cómo movilizar el potencial de la extensión agraria y rural? Roma: FAO.

Cortijo, E., Faure, G. y Le Gal P. Y. (2010). Inserción de las pequeñas explotaciones familiares en la cadena de suministro de los lácteos en el Valle del Mantaro: bacia una gestión de apoyo que tome en cuenta la diversidad de los actores.Montpellier: CIRAD.

Cristovao, A., Koutsouris, A. y Kügler, M. (2012). Extension systems and change facilitation for agricultural and rural development. In I. Darnhofer, D. Gibbon $\&$ B. Dedieu (Eds.). Farming systems research into the 2ist century: the new dynamic (pp. 20I-228), Dordrecht: Springer.

Dirección Regional de Agricultura de Junín (20II). Rec.04 de febrero de 2013. Retrieved from www.agrojunin.gob.pe

Dulcire, M. (2014). De la producción individual hacia un contrato colectivo: aprendizaje de los agricultores. Revista de ciencias sociales, 20(I), $7 \mathrm{I}-83$.

Faure, G., Desjeux, Y. y Gasselin, P. (20II). Revue bibliographique sur les recherches menées dans le monde sur conseil en agriculture. Cabiers Agricultures, 20(5), 327-342.

Fernández-Baca, E. y Bojorquez, C. (1994). Diagnóstico de la producción lechera en el valle del Mantaro. Revista de Investigación Pecuaria, 7(2), 97-106.

Gamboa, C. (2012). Producción y comercialización de la leche en el Valle del Mantaro, provincia de Concepción - Junín. Tesis de Lic. Economía, Lima: UNALM.

Goulet, F. (20II). Accompagner et vendre. Les firmes de l'agrofourniture dans l'innovation et le conseil en agriculture. Cabiers Agricultures, 20(5), 382-386. 
Gutiérrez, C. (2007). Modelo de tipificación de agencias agrarias. Lima: Ministerio de Agricultura.

Huamanyauri, M. K. (2013). Caracterización de los servicios de extensión of recidos a productores lecheros en la provincia de Concepción - Junín, Tesis de Lic. Zootecnia, Lima: UNALM.

Kidd, A. D., Lamers, J. P. A., Ficarelli, P. P. y Hoffmann, V. (2000). Privatising agricultural extension: caveat emptor. Journal of Rural Studies, I6, 95-102.

Klerkx, L., Grip, K. D. y Leeuwis, C. (2006). Hands off but strings attached: The contradictions of policy-induced demand-driven agricultural extension. Agriculture and Human Values, 23, I89-204.

Klerkx, L., Hall, A. y Leeuwis, C. (2009). Strenghtening agricultural innovation capacity: are innovation brokers the answer? International Journal of Agricultural Resources, Governance and Ecology, 8(5/6), 409-438.

Labarthe, P. (2005). Trajectoires d'innovation des services et inertie institutionnelle. Dynamique du conseil dans trois agricultures européennes. Géographie, Economie, Société, 73, 289-3II.

Landini, F. (20I2). Problemas en la extensión rural paraguaya: modelos de extensión en la encrucijada. Cuadernos de Desarrollo Rural, 9(69), I27-I49.

Laporte, M., Faure, G., y Le Gal, P. Y. (2008). Diversidad de las explotaciones agrícolas en los sistemas irrigados del valle del Mantaro y acceso de los productores al mercado. Montpellier: SupAgro, ENSAT, PSI, CIRAD.

Le Coq, J. F., Faure G. y Sáenz F. (2010). Las organizaciones de productores y las modalidades de prestación de los servicios agrícolas: lecciones de varios estudios de caso en Costa Rica. Revista Centroamericana de Ciencias Sociales, 7(2), 23-52.

Mirani, Z. D., Bukhari, S. S. y Narejo, M. A. (2007). Assessment of the impact of farm advisory services in Sanghar and Mirpurkhas districts of Sindh province of Pakistan. Agricultural Engineering, Veterinary Sciences, 23, 39-46.

Puente, J., Gallego, F. y Vidueira, P. (20II). La estructuración y dinamización social para una mejor gobernanza de las comunidades rurales. Cuadernos de Desarrollo Rural, 8 (66),73-IoI.

Rivera, W. M. (2000). Confronting global market: public sector agricultural extension reconsidered. Journal of extension systems, I6, 33-54.

Rivera, W. M. y Zijp, W. (2002). Contracting for agricultural extension: international case studies and emerging practices. Wallingford: CABI Publishing.

Röling, N. y Groot, A. (1998). Contemplating alternatives.A comparative framework for thinking about extension. Agriculture+Rural Development, 5, II-I3. 
Scoones, I. y Thompson, J. (eds) (2009). Farmer First revisited. Rugby: PracticalAction Publishing.

Vásquez, R. y Aguilar, I. (2010). Organizaciones lecheras en los Altos Sur de Jalisco: un análisis de las interacciones productivas. Región y Sociedad, 22(48), II3-I44. 